\title{
LOWER BOUNDS FOR THE SOLUTIONS IN THE SECOND CASE OF FERMAT'S LAST THEOREM
}

\author{
LE MAOHUA
}

(Communicated by William Adams)

\begin{abstract}
Let $p$ be an odd prime. In this paper, we prove that if $p \equiv 3$ $(\bmod 4)$ and $x, y, z$ are integers satisfying $x^{p}+y^{p}=z^{p}, p \mid x y z, 0<x<$ $y<z$, then $y>2^{-1 / p} p^{6 p-2}$ and $z-x>\frac{1}{2} p^{6 p-3}$.
\end{abstract}

Let $p$ be an odd prime. In [1] and [2], Inkeri showed that if $x, y, z$ are integers satisfying

$$
x^{p}+y^{p}=z^{p}, \quad \operatorname{gcd}(x, y)=1, p \mid x y z, 0<x<y<z,
$$

then $y>\frac{1}{2} p^{3 p-1}$ and $z-x>\left(2 p^{20 / 7}\right)^{p}$. In this paper, we prove the following result:

Theorem. If $p \equiv 3(\bmod 4)$ and $x, y, z$ are integers satisfying (1), then $y>$ $2^{-1 / p} p^{6 p-2}$ and $z-x>\frac{1}{2} p^{6 p-3}$.

Proof. It is a well-known fact that (1) is impossible for $p=3$, so we may assume that $p>3$. We first deal with the case that $p \mid z$. Let $\zeta$ be a $p$ th primitive root of 1 . Let $S$ be the set of nonzero squares $\bmod p$, and let $\theta=\prod_{i \in S}\left(x+y \zeta^{i}\right)$. Since $S$ represents $\operatorname{Gal}(\mathbb{Q}(\zeta) / \mathbb{Q}(\sqrt{-p}))$, it follows that $\theta$ is an algebraic integer in $\mathbb{Q}(\sqrt{-p})$, so $\theta=A+B \sqrt{-p}$, where $A, B \in \frac{1}{2} \mathbb{Z}$. Note that $\theta_{0}=\prod_{i \in S}\left(1-\zeta^{2}\right)$ is an algebraic integer in $\mathbb{Q}(\sqrt{-p})$ with norm $p$; hence, it must be $\pm \sqrt{-p}$. From

$$
\theta \equiv \begin{cases}\theta_{0}= \pm \sqrt{-p}(\bmod 2), & 2 \nmid x, 2 \nmid y, \\ \prod_{i \in S} 1=1(\bmod 2), & 2 \nmid x, 2 \mid y, \\ \prod_{i \in S} \zeta^{i}=1(\bmod 2), & 2 \mid x, 2 \nmid y,\end{cases}
$$

we find that $A+B \sqrt{-p}$ is congruent modulo 2 to an element of $\mathbb{Z}(\sqrt{-p})$, so $A, B \in \mathbb{Z}$. Further, since

$$
\theta \equiv \prod_{i \in S}\left(x-x \zeta^{i}\right)=0 \pm x^{(p-1) / 2} \sqrt{-p} \quad(\bmod x+y),
$$

Received by the editors November 11, 1989 and, in revised form, March 27, 1990.

1980 Mathematics Subject Classification (1985 Revision). Primary 11D41. 
we get

$$
A \equiv 0 \quad(\bmod x+y) .
$$

Let $p^{\alpha} \| z$. Classical relations due to Abel $[4$, p. 54] imply that

$$
x+y=p^{\alpha p-1} a^{p}, \quad \frac{x^{p}+y^{p}}{x+y}=p b^{p}, \quad z=p^{\alpha} a b,
$$

where $a, b$ are positive integers satisfying $p \nmid a b$ and $2 \nmid b$. From the above, we have $p b^{p}=A^{2}+p B^{2}$. Hence $p \mid A, p \nmid B$, and

$$
b^{p}=p A^{\prime 2}+B^{2}
$$

where $A^{\prime}=A / p$. By (2) and (3), we get

$$
A^{\prime} \equiv 0 \quad\left(\bmod p^{\alpha p-2}\right) \text {. }
$$

The factors $x+y \zeta^{i}$ are pairwise relatively prime for $1 \leq i \leq p-1$, except for factors of $1-\zeta$. Therefore $\prod_{i \in S}\left(x+y \zeta^{i}\right)=A+B \sqrt{-p}$ and $\prod_{i \notin S}\left(x+y \zeta^{i}\right)=$ $A-B \sqrt{-p}$ are relatively prime, except for primes above $p$. Recall that $p \nmid B$. We have $\operatorname{gcd}(A, B)=1$. It also follows that $A^{\prime} \sqrt{-p}+B$ and $A^{\prime} \sqrt{-p}-B$ are relatively prime in $\mathbb{Z}[\rho]$, where $\rho=(-1+\sqrt{-p}) / 2$. Hence they are $p$ th powers of ideals. Since the class number of $\mathbb{Z}[\rho]$ is less than $p$, it is prime to $p$. Therefore $A^{\prime} \sqrt{-p}+B$ is the $p$ th power of a number in $\mathbb{Z}[\rho]$. It implies that

$$
A^{\prime} \sqrt{-p}+B=\left(X_{1}+Y_{1} \sqrt{-p}\right)^{p},
$$

where $X_{1}, Y_{1} \in \frac{1}{2} \mathbb{Z}$. If $X_{1}+Y_{1} \sqrt{-p} \notin \mathbb{Z}(\sqrt{-p})$, then $X_{1}=u / 2, Y_{1}=v / 2$, with $u, v \in \mathbb{Z}$ and $u \equiv v \equiv 1(\bmod 2)$. By Waring's formula [3, Formula 1.76], we get from (4) and (6) that $u^{2}+p v^{2}=4 b$ and

$$
\begin{aligned}
2 B & =\left(\frac{u+v \sqrt{-p}}{2}\right)^{p}+\left(\frac{u-v \sqrt{-p}}{2}\right)^{p} \\
& =\sum_{j=0}^{(p-1) / 2}(-1)^{j} \frac{(p-j-1) ! p}{(p-2 j) ! j !} u^{p-2 j} b^{j} \equiv \sum_{j=0}^{(p-1) / 2}(-1)^{j} \frac{(p-j-1) ! p}{(p-2 j) ! j !} \\
& =\left(\frac{1+\sqrt{5}}{2}\right)^{p}+\left(\frac{1-\sqrt{5}}{2}\right)^{p}=L_{p} \quad(\bmod 2),
\end{aligned}
$$

where $L_{p}$ is the $p$ th Lucas number. Since $L_{p} \equiv 0(\bmod 2)$ only if $p=3$, it follows that $X_{1}+Y_{1} \sqrt{-p} \in \mathbb{Z}[\sqrt{-p}]$, and

$$
X_{1}^{2}+p Y_{1}^{2}=b, \quad X_{1}, Y_{1} \in \mathbb{Z} .
$$

We get from (6) that

(8) $A^{\prime}=\left(\begin{array}{c}p \\ 1\end{array}\right) X_{1}^{p-1} Y_{1}-\left(\begin{array}{c}p \\ 3\end{array}\right) p X_{1}^{p-3} Y_{1}^{3}+\cdots+(-1)^{(p-1) / 2}\left(\begin{array}{c}p \\ p\end{array}\right) p^{(p-1) / 2} Y_{1}^{p}$. 
Since $p \nmid b$, we have $p \nmid X_{1}$ by (7), and hence

$$
Y_{1} \equiv 0 \quad\left(\bmod p^{\alpha p-3}\right)
$$

by (5) and (8). If $Y_{1}=0$, then $A=0, B= \pm 1$, and $b=1$ by (4). Since $\left(x^{p}+y^{p}\right) /(x+y) \geq 2^{p-2}+1$, it is impossible that $p>3$. Therefore $Y_{1} \neq 0$ and $\left|Y_{1}\right|>p^{\alpha p-3}$ by (9), and $z=p^{\alpha} a b>p^{2 \alpha p+\alpha-5}$ by (7). Note that $\alpha \geq 3$, by [5]. We obtain $z>p^{6 p-2}$. Using the same method, we can prove that $y>p^{6 p-2}$ or $x>p^{6 p-2}$ correspond to $p \mid y$ or $p \mid x$. Thus $y>2^{-1 / p} p^{6 p-2}$ since $2^{1 / p} y>z$. Simultaneously, we have

$$
\begin{aligned}
z-x & =\frac{y^{p}}{z^{p-1}+x z^{p-2}+\cdots+x^{p-1}}>\frac{y^{p}}{p z^{p-1}}>\frac{y^{p}}{p\left(2^{1 / p} y\right)^{p-1}} \\
& =\frac{y}{2^{p-1 / p} p}>\frac{1}{2} p^{6 p-3} .
\end{aligned}
$$

The theorem is proved.

\section{ACKNOWLEDGMENT}

The author would like to thank the referee for his valuable suggestions.

\section{REFERENCES}

1. K. Inkeri, Abschätzungen für eventuelle Lösungen der Gleichung im Fermatschen Problem, Ann. Univ. Turku, 1953, 3-9.

2. __ Remarks on Fermat's equation: The very knowledge of coding, Univ. Turku, Turku, 1987, pp. 82-87.

3. R. Lidl and H. Niederreiter, Finite fields, Addison-Wesley, Reading, MA, 1983.

4. P. Ribenboim, 13 lectures on Fermat's last theorem, Springer-Verlag, New York, 1979.

5. H. Vandiver, A property of cyclotomic integers and its relation to Fermat's last theorem, Ann. of Math. 21 (1919), 73-80.

Research Department, Changsha Railway Institute, Changsha, Hunan, People's RePUBLIC OF CHINA 